\title{
Development of Consumer Preference based Demand Response Model for Home Energy Management
}

\author{
M. Krishna Paramathma, D. Devaraj,
}

\begin{abstract}
Demand response has become an effective method for energy saving and to reduce the energy cost. By adjusting the residential loads, it reacts quickly for the mismatches of supply and demand. In this paper, the Internet of Things (IoT) based Demand Response (Load scheduling method) methodology is proposed to mitigate the energy waste and tariff. Two different alarm provisions are made at the consumer side to indicate the normal and demand modes operated by the supplier. The consumer is provided with a controller that read the market signal and answers with consumer preferences. Whenever demand mode arises, the consumer is completely free to change the load setting; also the developed system will propose the load pattern. The demand mode will be given during peak hour and the tariff will be high at that time and normal mode will consist of the minimum tariff. The consumer may control their load through cloud-MQTT by giving a specific command or from the MQTT dashboard android app. Based on the tariff, the consumption profile could be reduced.
\end{abstract}

\section{Keywords: cloud mqtt, IoT, Demand response}

\section{INTRODUCTION}

A perfect balance between supply and demand is needed to ensure the reliable operation of electricity system. Maintaining the balanced condition irrespective of generation unit forced outages, transmission and distribution line outages, and sudden load changes is a challenging task. Smart Grid is one of the major innovations of electric energy management system, which amalgamate communication infrastructure with control and sensor technology [1].
Further, DR is classified as incentive based programmes and price based programmes. It helps to facilitate the efficient management of load demand variation by interconnecting all the consumers [2]. At distribution level, this variation of load demand is made possible by demand response [DR]. It is denoted as change in the way of electricity utilization according to the electricity price [3].

The former is based on curtailing the consumers load on a specified agreed level and later is based on real time price variation over the period [4]. The DR system includes the AMI, load controller scheduling manager and database as shown in Figure 1. The Advanced Metering Infrastructure (AMI) allows for two-way communication between a residential consumer and the retailer. The load controller links home appliances and the demand response unit. After receiving the scheduling list from the scheduling manager, the load controller can operate each appliance. The consumer can monitor the scheduling results by using a smart phone

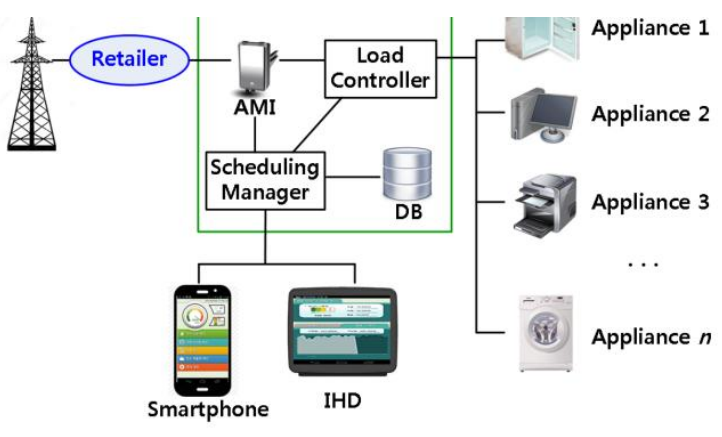

Figure 1: Demand Response System[5]

\section{LITERATURE REVIEW}

Many budding researches have been sprouted in recent years from United States and European countries on residential DR [6]. Gyamfi and others [7] outlined some of the residential DR behavioral problems to investigate customer response for various DR programs, including Time of Use and Real Time Pricing strategies. The domestic response of customers was studied by Hamidi et al.[8] in DR programs.

The purpose of this study is to show the impact on responsiveness of various tariffs. Yoon et al. [9] suggested a DR plan for a residential building heating, ventilation and air conditioning (HVAC) system based on dynamic energy pricing to ensure a certain level of comfort for the consumer.

Revised Manuscript Rec
* Correspondence Author

M. Krishna Paramathma, Electrical and Electronics Engineering, Kalasalingam Academy of Research and Education, Anand Nagar, Krishnankoil, Virudhunagar, India, paramathma.klu@gmail.com

D. Devaraj, Electrical and Electronics Engineering, Kalasalingam Academy of Research and Education, Anand Nagar,
Residential loads are categorized as elastic, inelastic, 
common and thermostat controlled loads [10].

The adopted method is suitable only in smart homes. A priority based scheduling is deployed to decide suitable load in a particular time is proposed in[11].

Raspberry Pi 3 model B based Home Energy Management System (HEMS) is proposed in [12]. The load has to be turned on and off consequently for the system to determine the suitable algorithm.

To overcome the above stated issues, our proposed system deals with a simple and efficient DR scheme which suggests consumer to turn ON/OFF the suitable load based on the demand. It also indicates the consumer when there is demand mode and the control of load through IoT.

\section{Proposed methodology}

Our proposed system focuses on consumer sophistication and efficient energy usage as main priority. Energy loss also occurs due to improper usage of energy received. The proposed system will allow the consumer to view their energy usage and also the tariff. The system not controls the load but will suggest the consumer to do so but the final decision is on the consumer's hand.

The energy consumption is constantly monitored and displayed to the consumer. The electricity board will notify when there is a demand mode i.e., peak hour. The tariff for the demand mode will also be notified and displayed.

IoT is used by the electricity board to notify the consumers. The load can also be controlled by the consumer using the same IoT platform. The load can also be controlled by using cloud mqtt app that's suitable with android which provides the consumers an easy way to control the load.

The proper usage of the received energy is the way to achieve an efficient energy use. This method is also suitable for normal houses rather than smart houses. Since IoT is used it provides ease of access to the consumer, and also to the electricity board.

\section{METHODOLGY}

The schematic diagram of the project is given below in the figure 1.

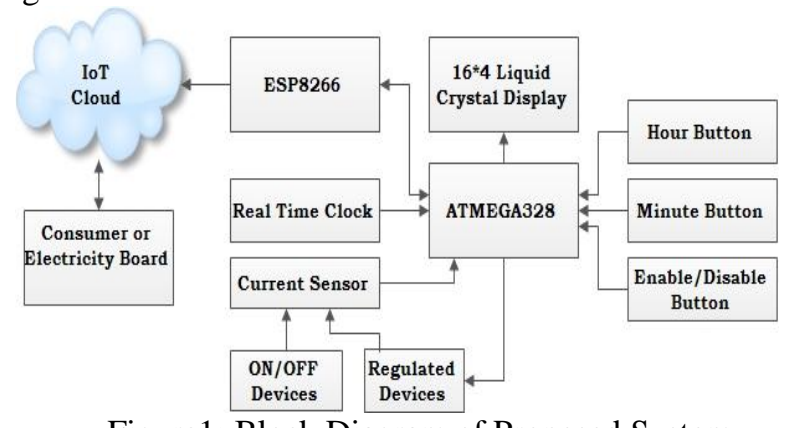

Figure1: Block Diagram of Proposed System.

\author{
2. ATMEGA 328 controller \\ 3. MQTT IoT cloud \\ 4. Relay modules \\ 5. Home appliances(load)
}

The proposed Smart Home setup consists of RTC DS1307 module, current sensor, ATMEGA328 controller, ESP8266 module and other devices like, relay, LCD module and buttons. DS1307 RTC module has Vcc, Ground, Serial data (SDA), and Serial clock (SCL). Vcc and Ground pins are connected to $5 \mathrm{~V}$ and Ground pins of controller respectively that gives the biasing voltage for the operation. SCL and SDA pins are connected to ATMEGA328 SCL and SDA pins. The controller in-turn communicates with RTC DS1307 using these two pins. Three buttons are connected to ATMEGA328 digital pins 2, 3 and 4. All three push buttons are pulled down to ground through $10 \mathrm{~K}$ resistors as shown. When any button is pressed, the respective controller pin gets logic $1(\mathrm{HIGH})$ input. Relay modules are used to turn ON/OFF the devices. The current and voltage sensors are interfaced with Controller; the gathered parameters will be sending to IoT cloud through ESP8266. LCD display is kept for consumer's conveniences.

CloudMQTT are managed Mosquitto servers in the cloud. Mosquitto implements the MQ Telemetry Transport protocol, MQTT, which provides lightweight methods of carrying out messaging using a publish/subscribe message queueing model. MQTT is the machine-to-machine protocol of the future. It is ideal for the "Internet of Things" world of connected devices. Its minimal design makes it perfect for built-in systems, mobile phones and other memory and bandwidth sensitive applications.

Message queues provide an asynchronous communications protocol, the sender and receiver of the message do not need to interact with the message queue at the same time. Messages placed onto the queue are stored until the recipient retrieves them or until the messages times out. MQTT and Mosquitto are for good use by bandwidth sensitive applications. Cloud MQTT let you focus on the application instead of spending time on scaling the broker or patching the platform.

\section{RESULT AND DISCUSSION}

The entire hardware connection is shown in the Figure (2).

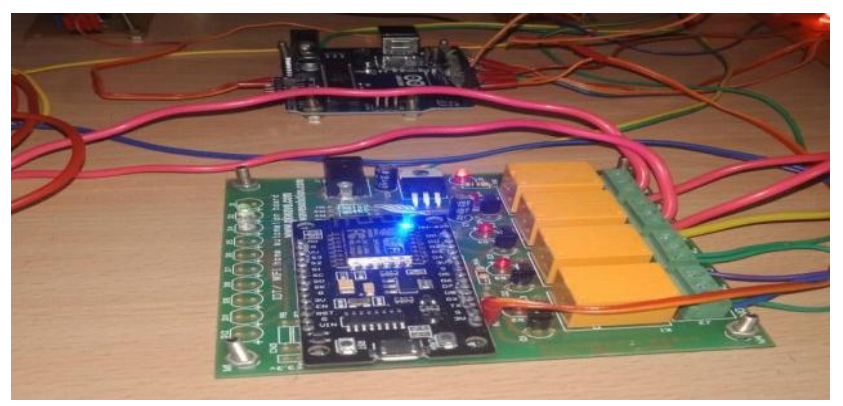

Figure 2: Node MCU with Relay kit.

The components which are used in this proposed work are mentioned below;

\section{Node MCU}




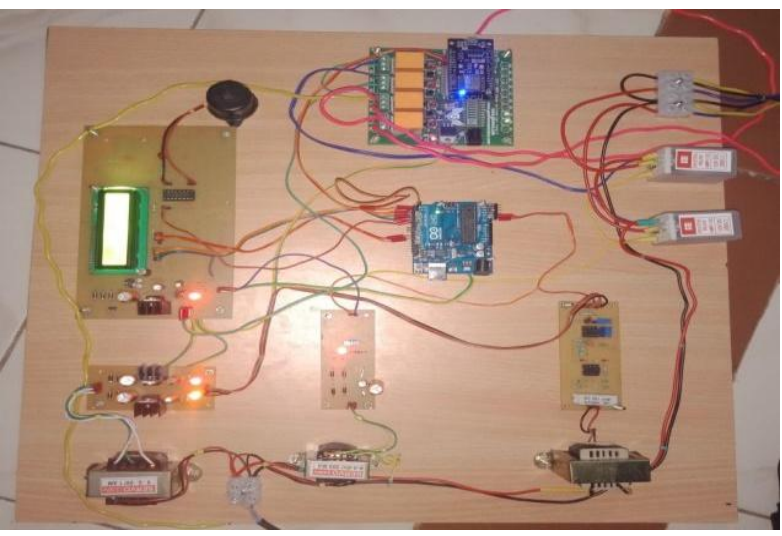

Figure3: Hardware connection.

The setup will be connected to the internet through node-mcu. The values from current sensing circuit and voltage sensing circuit are displayed in the LCD. The cloud mqtt page from which the commands were given is shown in figure (3)

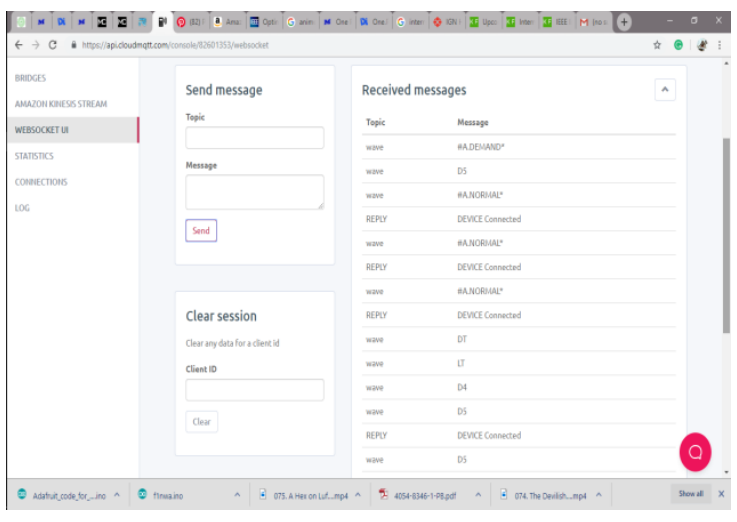

Figure 4: cloud mqtt page

The following figures 4 and 5 shows the system in normal and demand mode which is displayed in LCD. The image shown here shows all loads in off condition and the voltage value will be visible.

From the send message field in the figure 3 the commands can be given. The commands to control the loads are L5, L6, L7\&L8 to turn the load 1, 2, 3, 4 ON and the commands D5, D6, D7\&D8 are used to turn the loads off. To turn all the load on command LT will be used and to off the load was DT.

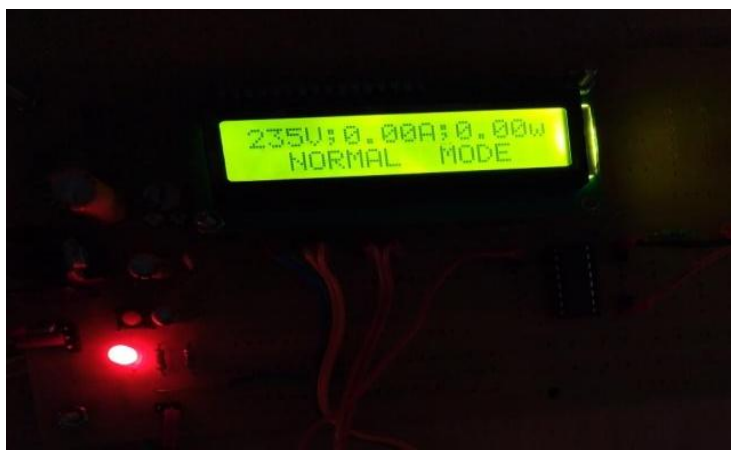

Figure5: LCD Display (Normal Mode).

The above figure shows the LCD showing that the system is in normal mode and the consumer can turn any load on as the tariff is normal in this system.

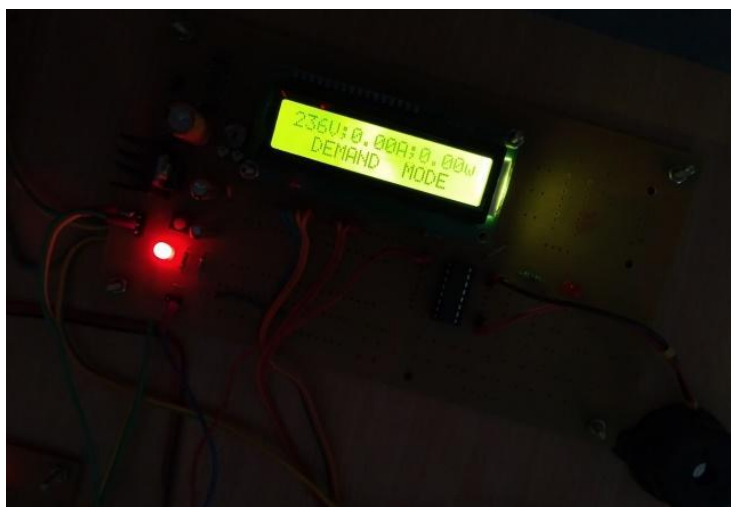

Figure6: LCD Display (Demand Mode).

The above figure shows system in demand mode i.e., peak time and the tariff is high. So the consumer has to turn heavy loads off in order to reduce their bill and to reduce strain in the system during peak hour.

The relays connected to the node mcu will control the load connected to the circuit. The node mcu can also control the load off line when the user is not connected with the cloud. In other words when the kit is off line it'll work as home automation kit and while online it can be used to control the load through IoT based on the energy mode.

Ultimately the control relies on hands of consumers, which means that everyone should focus on conserving energy in order to achieve less energy loss and efficient usage of energy. The consumers should also learn to schedule their heavy loads during normal hours to reduce the strain in system and for efficient usage

\section{CONCLUSION}

Natural resources are the cause of 90 percent electricity production and are exhausted on a large scale day by day. It is, therefore, the duty of people to use electricity efficiently and effectively to save natural resources for the next generation. This article provided a straightforward and effective DR methodology for scheduling residential loads. The suggested scheme also offers the customer with the sophistication to regulate the loads through IoT cloud and mqtt apps. This development could lead a society with maximum energy usage efficiency and a better way to use the residential loads

\section{REFERENCES}

1. Muhammad Babar Rasheed, Nadeem Javaid, Muhammad Awais, Zahoor Ali Khan ,Umar Qasim , Nabil Alrajeh, Zafar Iqbal and Qaisar Javaid "Real Time Information Based Energy Management Using Customer Preferences and Dynamic Pricing in Smart Homes", 2016.

2. Asha Radhakrishnan M.P. Selvan "Load Scheduling For Smart Energy Management in Residential Buildings with Renewable Sources", 2014.

3. Muhammad Umer Qureshi,AlanGirault, Mickael Mauger ; Santiago Grijalva, "Implementation of home energy management system with optimal load scheduling based on real-time electricity pricing models", 2017 IEEE 7th International Conference on Consumer Electronics - Berlin (ICCE-Berlin)

4. Syed Qaseem Ali S. Danish Maqbool Imthias Ahamed Imthias Ahamed, "Load scheduling with maximum demand and time of use pricing for microgrids ",2013 IEEE Global Humanitarian Technology Conference 
5. Park, L.; Jang, Y.; Bae, H.; Lee, J.; Park, C.Y.; Cho, S. Automated Energy Scheduling Algorithms for Residential Demand Response Systems. Energies 2017, 10, 1326.

6. Ryuto Shigenobu ; Mitsuki Sagara; Atsushi Yona; Tomonobu Senjyu, "Optimal scheduling of real-time pricing electrical power market considering participation rate of demand response" 2015 IEEE 2nd International Future Energy Electronics Conference (IFEEC).

7. Gyamfi S, KrumdieckS, UrmeeT. Residential peak electricity demand response-highlights of some behavioral issues. Renew Sustain Energy Reviews 2013;25: 71-7.

8. Hamidi V, LiF, Robinson F. Demand response in the UK's domestic sector. Electrical Power System Res 2009;79:1722-6.

9. Yoon JH, Bladick R, Novoselac A. Demand response for residential buildings based on dynamic price of electricity.EnergyBuild 2014;80:531-41.

10. Zhanle Wang; Raman Paranjape "Optimal Residential Demand Response for Multiple Heterogeneous Homes With Real-Time Price Prediction in a Multiagent Framework 'IEEE Transactions on Smart Grid Volume: 8 , Issue: 3 , May 2017.

11. H. Fraser, "The importance of an active demand side in the electricity industry," The Electricity Journal, Elsevier, Volume 14, Issue 9, pp. 5273, November 2001.

12. G. Strbac, "Demand side management: Benefits and challenges," Energy Policy, Elsevier, Volume 36, Issue 12, pp. 4419-4426, December 2008.

\section{AUTHORS PROFILE}

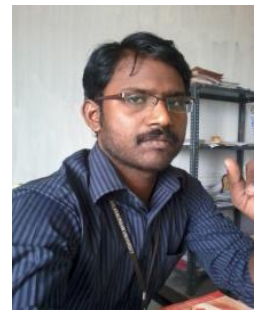

Krishna Paramathma has received his B.E and M.E degree in Electrical and Electronics Engineering and Power System Engineering in 2007 and 2010, respectively, from Anna University and Kalasalingam Academy of Research and Education. He is currently working as Assistant Professor in the department of Electrical and Electronics Engineering, Kalasalingam Academy of Research and Education Krishnankoil, India.

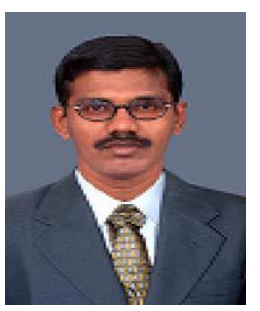

D. Devaraj has received his B.E and M.E degree in Electrical and Electronics Engineering and Power System Engineering in 1992 and 1994, respectively, from Madurai Kamaraj University, Madurai, India. From 1994to1997, he worked as a Lecturer in Arulmigu Kalasalingam College of Engineering, Krishnankoil. He received his Ph.D. degree from IIT Madras, Chennai, in 2001. He is currently a Professor and Head of the Electrical and Electronics Engineering Department in Kalasalingam Academy of Research and Education Krishnankoil, India. His research interest includes Applications of Computational Intelligent Techniques, Power System Optimization, Power System Security, Fault Diagnosis and Data Mining. 